\title{
Library Management and Access System Using Bluetooth
}

\author{
SarkarTanvir $^{1}$, Kundu Subhajit ${ }^{2}$ \\ ${ }^{1,2,}$ ESL, Chandannagar Lab CA-5 Salt Lake City, Kolkata - 64
}

\begin{abstract}
In this project we developed a Bluetooth based library management \& access system coded in python programming language.The methodology section describes the design and implementation of the proposed system in details. The system provides a user friendly and convenient way to access any library in a very less hectic manner. It provides user a magnificent platform to navigate through huge stockpile of books in an easy digital format with help of a "client-server protocol" using Bluetooth technology. The system navigates the search of a user for his/ her desired book by categorizing it in three major divisions; i)search by topic ii)search by book iii)search by author, thus making the search a time efficient and convenient one and also supplying the user with the perfect match for his search.
\end{abstract}

Keywords: Bluetooth, Library Management System, Python, Server Client protocol.

\section{Introduction}

We aim to develop this system which can smoothen the job of finding the required book for a user reducing his manual labor, time of search but also a perfect match for his search with all the necessary details, and simultaneously providing the librarians a flexible database to keep an account of each and every book according to their name, author's name, quantity, and rack no. Along with all this we are strongly determined to develop a better and efficient interface between library and it's user with this system. But the most crucial point is that we are providing all the facilities with the help of very cheap and widely available technologies, which makes us distinct from others.

We have implemented the following technologies in this project:

- Bluetooth(as a mode of communication)

- Python(as coding language)

\subsection{What is Python?}

- Python is a general-purpose, high-level programming language whose design philosophy emphasizes code readability. Python language stands out in comparison with respect to other programing languages as it is compact.

- Can be packaged into standalone executable file which can cater to our several needs by using third party tools.

- Provides a simpler and better way to represent data in graphical form.

- Modules like Bluetooth, time, Os, Tkinter provides us with the facility to construct a suitable server client protocol for the users develop suitable GUI for the users, track the different Mac ID's of the Bluetooth's.

- Is an interpreted language that allows for rapid, flexible, exploratory software development.

1.2 Why python?

Python language stands out in comparison with respect to other programing languages in terms of its vast array of standard library and code readability. We chose it over other languages as it is more compact and also by using third party tools python code can be packaged into standalone executable file which can cater to our several needs. We have used third party modules like Pybluez, Tkinter. Pybluez module helps us to perform the scanning and tracking operation through bluetooth and to construct an easy, convenient and simple GUI we here used the module Tkinter.

\subsection{What is Bluetooth?}

Bluetooth is a wireless technology standard for exchanging data over a short distance in a very efficient and lucid way. It is a technology standard for exchanging data over short distances (using short wavelength radio transmissions in the ISM band from $(2400-2480 \mathrm{MHz})$ from fixed and mobile devices, creating personal area network (PANs) with high levels of security. 


\subsection{Why Bluetooth?}

- It is very cheap and easily available. A Bluetooth adapter costs very less (around INR 200) and can be found easily in the market. Therefore its maintenance can be done easily.

- It can connect several devices, overcoming problems of synchronization.

- It is very easy to install, unlike its other connecting devices it consumes very less power so it can operate without an external source.

- Limited area of access proves to be quite an advantage for our project.

- Does not interfere with normal signaling of devices.

- Since our project deals with managing the database of any library and again using that same database to accomplish the search of number of user, we used Bluetooth as a medium to transmit information because it can avoid the interference of an external device.

\section{Motivation}

We are quite well aware of the fact in this modern era of $21^{\text {st }}$ century library is a highly important and invincible part of our civilized society. To expand knowledge and education in all aspect of our society on a huge basis we cannot ignore the importance of library. Though in modern time we have all sorts of access to study materials and literature through internet but libraries still serve the purpose of expanding, enhancing and quenching one's thirst for knowledge. Though we having such a lot of merits and utility for the society but we developed this system while we encountered some real life inconvenience while using our own college library. We often found the task of searching any book we required hectic, time consuming and manual so we decided to make the job easy by incorporating some technical touch to this entire process by building this 'access and management system'. Our system is aimed not only to lessen time consumption of a user to achieve the desired book but we also believe that the system will ease the job of the librarian to maintain the database.

\section{Methodology}

In the following section we are going to describe the methodology of the project we have done. We have divided our task in three major sections. The methodology of all the three sections are described below. 3.1 The Client side: In the client side i.e. the user side after running the particular codes finds four options. Three option to search and one option to exit. The options to search are categorized as

- Search by topic

- Search by book name

- Search by author

In case of search by topic if the user is unknown about the collection of books on a particular subject the user can search his/her desired book with on basis of the corresponding topic the book is associated with such as C, Microprocessor, Data Structure etc. when the user uses the category "search by book name". He/she can entry a particular book name to search; and in the third category the user can find or search all the books composed by any particular author. After choosing the convenient category and filling it with the necessary details the user press the send button on the GUI. The details are send in form of string and to send this information to the server the client side uses a particular block of code known as the client protocol. To send all this information the medium we here use Bluetooth.

3.2 The server side: The server installed in the library after receiving the information from different clients; the library sever searches in response to the request of the user in in its pre-defined dictionary. Now we must mention here that the data structure we used in this project to store the data about the books is a dictionary but the same purpose we can also serve by creating a data base. We here created a text file named "book.txt" which actually is a dictionary containing four values against each key, as the name of the author, the name of the book, the rack number of the book, finally the quantity of the book. After assembling all the necessary data in response to the search of the user, the server sends the accurate result via bluetooth. While sending the results back to the corresponding users the library server runs block of codes to send it via bluetooth.

3.3 Modification of the dictionary: This section deals with the upgradation of data in the dictionary in the text file "book.txt". When this block of code runs a GUI panel creates where there are three options "entry data", "exit", "update". After pressing the button to entry data he/she finds a new GUI with five entry boxes regarding the subject, book author, rack, quantity. After filling all the entry fields there is a "save" \& "exit" button. Exit to return to the previous GUI and pressing the save button one can again return to the previous GUI but entries are now saved. Now he/she just have to press the update button and the modified data shall be saved in the dictionary. 


\subsection{Flow Diagram:}

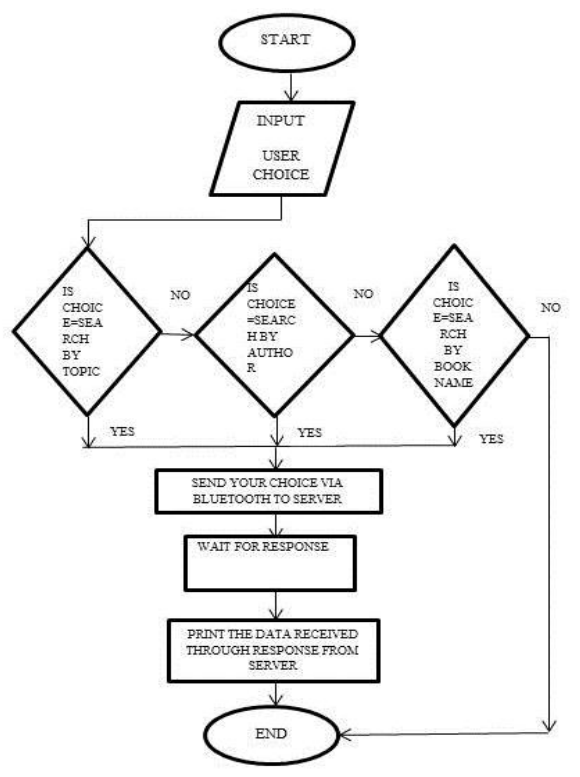

Fig 1: Flow diagram of the client side programme

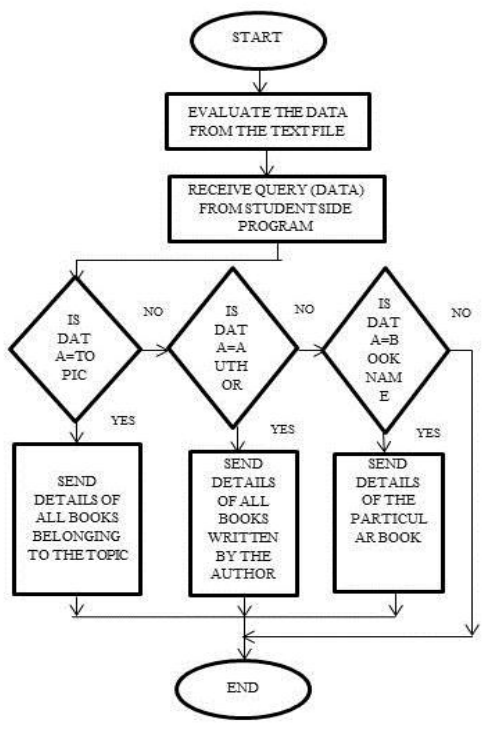

Fig 2: Flow diagram of the server side programme

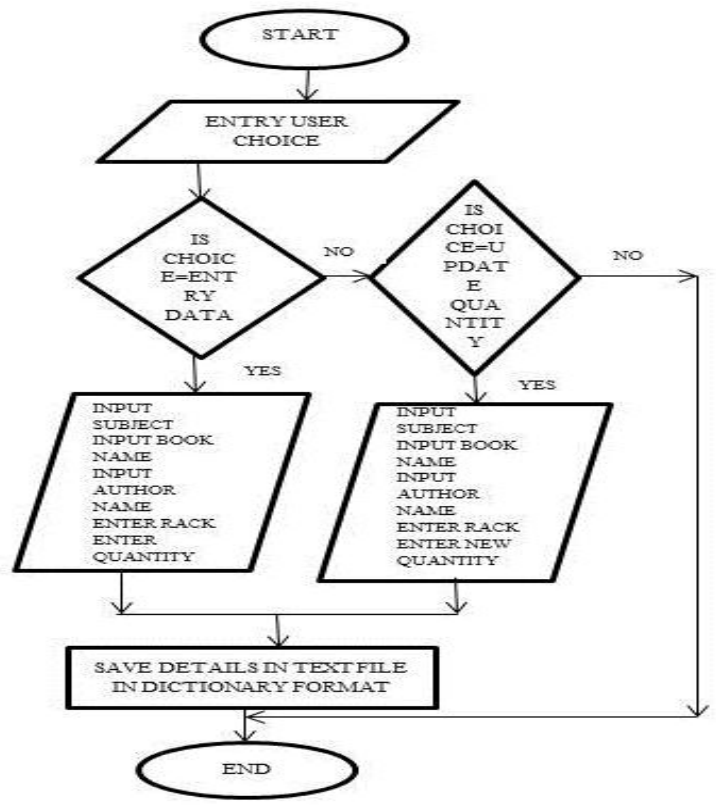

Fig 3: Flow diagram to modify the dictionary

- CLIENT SIDE PROGRAM:

IV. Algorithms

STEP 1: Start.

STEP 2: Input user choice.

STEP 3: If choice=search by topic then go to step 6. Else go to step 4 .

STEP 4: If choice=search by author then go to step 6. Else go to step 5 .

STEP 5: If choice=search by book name then go to step 6. Else go to step 9 .

STEP 6: Send choice via Bluetooth to the server.

STEP 7: Wait till the server responds according to the choice.

STEP 8: Print data received through response from server.

STEP 9: End.

- SERVER SIDE PROGRAM:

STEP 1: Start. 
STEP 2: Evaluate data from the text file.

STEP 3: Receive query from the client side.

STEP 4: If data=topic then go to step 5. Else go to step 6.

STEP 5: Send details of all the books belonging to the topic.

STEP 6: If data=author then go to step 7. Else go to step 8 .

STEP 7: Send details of all the books written by the author.

STEP 8: If data=book name then go to step 9. Else go to step 10.

STEP 9: Send details of the particular book.

STEP 10: End.

- DICTONARY MODIFICATION PROGRAM:

STEP 1: Start.

STEP 2: Entry user choice.

STEP 4: If choice=entry new data then go to step 5. Else go to step 6.

STEP 5: Input subject, book name, author name, rack no, quantity. After that go to step 8.

STEP 6: If data=update quantity then go to step 7. Else go to step 9.

STEP 7: Input subject, book name, author name, rack no, new quantity. After that go to step 8.

STEP 8: Save details in the text file in dictionary format.

STEP 9: End.

\section{Results}

\section{3.:Python26lpython.exe}

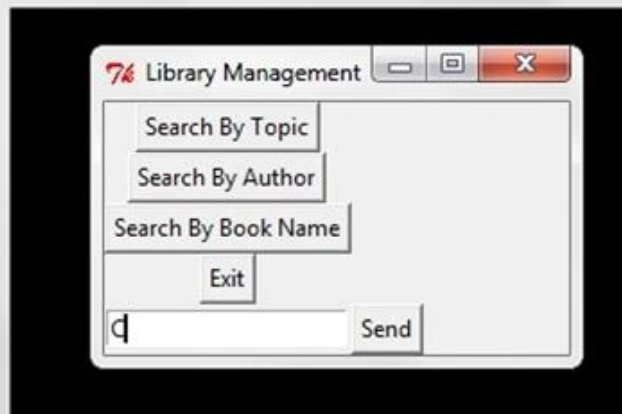

Fig 4: Request submission screen for user

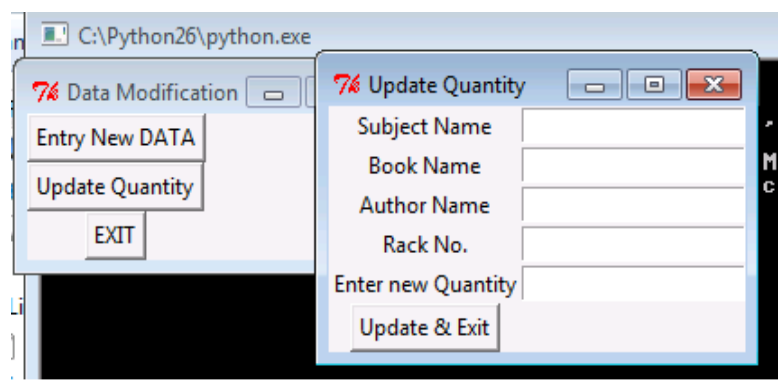

Fig 5: Screen to update the quantity of books

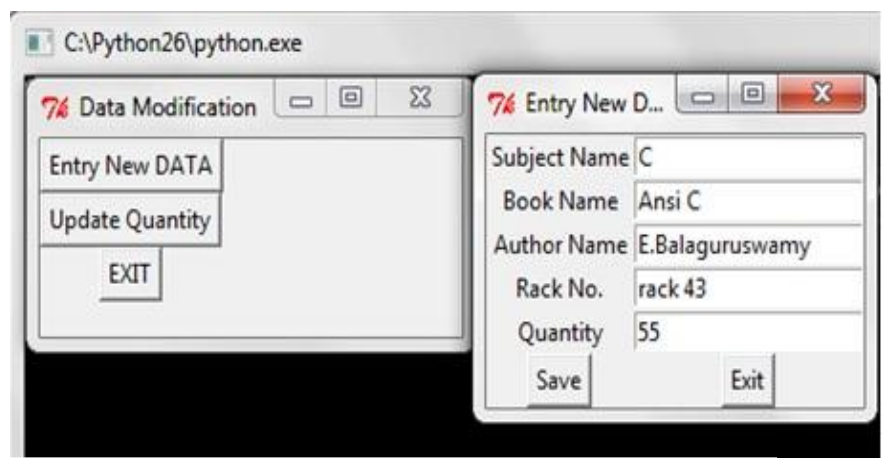

Fig 6: Screen to entry new data. 
In near future we are very much keen to develop the entire system by introducing whole new features and expanding our system from library to other different sectors such as shopping malls, departmental stores. We aim to upgrade the system and incorporate some new feature such as

i) While searching books on a certain topic the user will be notified with the most popular and renowned books on that very topic.

ii) The user shall be notified and alerted with if he/she has some dues in the library.

iii) The user shall be notified about the fresh arrived stocks of books on any certain topic.

iv) The user shall be given the cover page picture of the book he/she searched.

\section{Conclusion}

From a proper analysis of positive points and constraints of the system it is inferred that the system is working as per the objectives of the project. Installation and maintenance of the system is cost effective and takes less time. The system user interface is user friendly and does not require specialized training or skills to operate it. The project has been designed to substantially enhance the performance by ensuring a smooth search of books and easy management of library database. The implementation of the algorithm is done in such a way that it not only smoothen the search of an user by classifying the search in three different categories but also saves the valuable time of an user by turning the entire process into a highly time efficient, convenient one with most accurate and perfect match in result of the search. The system also reduces the workload of librarian as the up gradation of the database is quite easy. The result for any search from the user is totally automated with highest accuracy greatly reducing the domain of error.

\section{References}

[1] Mainak Samanta, Ankur Bhattacharya, Koushik Ghosh, Ritidrita Dhara, "Bluetooth Based Airport Enquiry System Using Python", International Journal of Scientific and Research Publications, Volume 3, Issue 9, September 2013.

[2] Avranil Tah, "A deadline-driven epidemic data collection protocol suitable for tracking interpersonal rendezvous" (January 1, 2010). Collection for University of Texas, El Paso. PaperAAI1483985. http://digitalcommons.utep.edu/dissertations/AAI1483985

[3] Nagadeepa N, "Enhanced Bluetooth Technology to Assist the High Way Vehicle Drivers", Research Journal of Recent Sciences, ISCA, Vol. 1(8), 82-85, August (2012).

[4] Ronojoy Ghosh, Vivek Shah, Hitesh Agarwal, Ashutosh Bhushan, Prasun Kanti Ghosh, "Intelligent ambulance traffic assistance system”, International Journal of Advanced Computer Science and Applications, Vol. 4, No. 8, 2013.

[5] Naveen Ersala, David C. Yen, "Bluetooth technology: a strategic analysis of its role in global 3G wireless communication era", Computer Standards \& Interfaces 24 (2002) 193 - 206

[6] Devbhuti Shounak, Ganguly Debojyoti, Majumder Debdeep, Payra Ritwik, "A Method for Bypassing Keystroke Recognition Based Security System Using Social Engineering", IOSR Journal of Computer Engineering (IOSR-JCE), IOSR, Volume 16, ISSue 2, Ver. II (Mar-Apr. 2014), PP 87-93. 